\title{
Fracture Resistance of Endodontically Treated Teeth Restored with Fiber Posts Luted with Composite Core Materials
}

\author{
Abdullah S Alshahrani ${ }^{1}$, Hassan B Alamri ${ }^{2}$, Faisal M Nadrah ${ }^{3}$, Moteb K Almotire ${ }^{4}$, Ahmed Y Alateeq ${ }^{5}$, Ibraheem F Alshiddi ${ }^{6}$, \\ Sayed R Habib ${ }^{7}$, Ala'a I Abu-Obaid ${ }^{8}$
}

\begin{abstract}
Aim: This study aimed to investigate the influence of using dual-cure composite core materials for fiber post luting on the fracture resistance of endodontically treated maxillary central incisors.

Materials and methods: Fifty sound and recently extracted maxillary central incisors were endodontically treated and distributed into five groups. In the control group, access cavities were restored with the composite resin. Experimental groups had the coronal structure trimmed $2 \mathrm{~mm}$ above the cementoenamel junction. Teeth were restored with fiber posts and different combinations of luting and core materials: RelyX Unicem for post luting and MultiCore for core buildup; MultiCore for post luting and core buildup; RelyX Unicem for post luting and LuxaCore for core buildup; or LuxaCore for post luting and core buildup. All teeth were restored with zirconium crowns and subjected to thermocycling $\left(6000\right.$ cycles $5 / 55^{\circ} \mathrm{C}$ ). Loading of the teeth was applied at $135^{\circ}$ to the palatal surface, using a universal testing machine at a cross-speed of 0.5 $\mathrm{mm} /$ minute until failure. The mode of failure was analyzed under a digital microscope.

Results: The fracture resistance of the control group was significantly higher than the experimental groups $(p<0.05)$. There was no significant difference between the experimental groups $(p>0.05)$. All teeth demonstrated nonrestorable failures.

Conclusion: With the presence of the ferrule effect and crown restoration, the selected materials for post luting and core buildup have no significant influence on the fracture resistance of the endodontically treated teeth.

Clinical significance: The use of the same dual-cure composite core materials for fiber post luting and core buildup would simplify the clinical procedure without enhancement of the fracture resistance of the endodontically treated tooth.

Keywords: Core buildup, Fiber post, Fracture resistance, In vitro study, Secondary monobloc, Self-adhesive resin cements.

The Journal of Contemporary Dental Practice (2020): 10.5005/jp-journals-10024-2814
\end{abstract}

\section{INTRODUCTION}

The success and longevity of endodontically treated teeth (ETT) primarily depend on the quality of endodontic treatment, remaining tooth structure, and coronal restoration. ${ }^{1}$ The deficiency in the tooth structure due to caries, trauma, pulpal pathosis, internal resorption, overpreparation, or developmental anomalies could result in a compromised remaining tooth structure that is susceptible to fracture under masticatory forces. ${ }^{2-6}$ Restoring such teeth is more challenging; therefore, the selection of a suitable post and core system that could dissipate occlusal forces to the tooth and its surrounding tissues is crucial to avoid root fracture. ${ }^{7}$ Different types of post and core systems, such as cast or prefabricated, metallic and nonmetallic posts, are commonly used for the restoration of ETT to provide the extracoronal restorations with retention and resistance forms. ${ }^{8}$

Metal posts with high rigidity could resist lateral forces, but they also transfer stresses to the less rigid dentin walls; such performance has been associated with a higher incidence of root fractures and unfavorable failures in comparison to fiber post and composite core systems. ${ }^{9}$ Fiber posts and composite cores for restoring ETT in the esthetic zone have been considered the preferred materials for several reasons, such as clinical convenience, single-visit procedure, ability to bond to the tooth structure and composite resin, esthetics, and cost-effectiveness. ${ }^{10,11}$ Fiber posts have the ability to flex under load and dissipate stresses to the surrounding tissues due to their modulus of elasticity matching the dentin. ${ }^{12-15}$ Different in vitro studies have demonstrated better fracture resistance and fracture mode with fiber posts than metal-based post systems. ${ }^{13,16-19} \mathrm{~A}$ higher
${ }^{1,2,6-8}$ Prosthetic Dental Sciences Department, College of Dentistry, King Saud University, Riyadh, Kingdom of Saudi Arabia

${ }^{3-5}$ College of Dentistry, King Saud University, Riyadh, Kingdom of Saudi Arabia

Corresponding Author: Abdullah S Alshahrani, Prosthetic Dental Sciences Department, College of Dentistry, King Saud University, Riyadh, Kingdom of Saudi Arabia, Phone: +966 554744778, e-mail: asalshahrani@ksu.edu.sa

How to cite this article: Alshahrani AS, Alamri HB, Nadrah FM, et al. Fracture Resistance of Endodontically Treated Teeth Restored with Fiber Posts Luted with Composite Core Materials. J Contemp Dent Pract 2020;21(4):383-389.

Source of support: Nil

Conflict of interest: None

rate of failure was found in teeth with cast posts and cores than with fiber posts and composite cores over 4 years of clinical evaluation. ${ }^{20}$

Studies reporting the best technique for restoring ETT to enhance fracture resistance are still controversial. ${ }^{21}$ The concept of a tooth-post-core monobloc in ETT was applied, first using carbon fiber posts, in 1996, and was based on the achievement of a single biomechanical complex by bonding all involved materials in the post-and-core system to one another and to the tooth through the use of materials with, ultimately, a modulus of elasticity similar to the dentin. ${ }^{22}$ This concept has been characterized as a secondary monobloc. ${ }^{23}$ 
Achieving a reliable bond between the post- and the intraradicular and coronal dentin is essential to attain a long-term success. ${ }^{7,24}$ Two adhesive methods are available for fiber post luting, with either a self-etch or an etch-and-rinse method; the self- or dual-curing resin cements are the most commonly used due to limited accessibility of light at the deeper level of the post space. ${ }^{25}$ In addition, self-adhesive resin cement is a dual-cure and a single-step material. ${ }^{26}$ Therefore, it is less technique-sensitive and suitable for luting of the fiber posts. ${ }^{24,25,27}$

Different composite resins with different filler amounts and types are used with fiber posts. They have a modulus of elasticity almost lower than the dentin, allowing them to act as a stressdumping interface between the post and the dentin. ${ }^{28}$ The conventional method is luting the fiber post with resin cement and core buildup with the composite resin core material. However, the availability of core materials with low viscosities permits their use as luting agents. ${ }^{10,28,29}$ Using the same material for post luting and core buildup is a time-saving procedure and has been advocated by several studies. ${ }^{30-32}$

A limited number of studies have been conducted on the fracture resistance of ETT restored using the same material for luting of the fiber posts and core buildup. The aim of this study was to investigate the effect of using the same composite resin material for fiber post luting and core buildup on the fracture resistance of endodontically treated maxillary central incisors restored with zirconium crowns. The null hypothesis was that there is no difference among the materials used for post luting and core buildup on the fracture resistance of endodontically treated maxillary central incisors.

\section{Materials and Methods}

\section{Specimen Preparation}

This study was approved by the King Saud University Institutional Review Board (E-17-2649). Fifty recently extracted maxillary central incisors due to periodontal diseases were used in this in vitro study. The inclusion criteria were sound teeth with no carious lesion, straight roots, closed apices, and lack of root cracks or previous endodontic treatment. The selected teeth were scanned under a stereoscopic microscope at $10 \times$ magnification to confirm the lack of cracks. Periapical radiographs were obtained to ensure the lack of internal resorption or canal calcification. Any deposits and soft tissue on the root surfaces were removed using an ultrasonic scaler. The length of the crown and the root as well as the faciopalatal and mesiodistal width were measured at the cementoenamel junction (CEJ) with a digital caliper (Mitutoyo, Kawasaki, Japan). ${ }^{33}$ The crown length was confined to $10 \pm 1.75 \mathrm{~mm}$, and the root length was confined to $13 \pm 1.75 \mathrm{~mm}$. The faciopalatal and mesiodistal width at CEJ was confined to $6.75 \pm 0.25 \mathrm{~mm}$ and $6.25 \pm 0.25 \mathrm{~mm}$, respectively. Afterward, the teeth were immersed for 5 minutes in $5.25 \%$ sodium hypochlorite $(\mathrm{NaOCl})$ solution and kept in saline solution at room temperature until use. One investigator performed the procedure of specimen preparation.

\section{Endodontic Treatment and Periodontal Ligament Simulation}

Before embedding the teeth in acrylic resin, they were dipped in melted wax $2 \mathrm{~mm}$ below the CEJ. A dental surveyor (Dentsply Neytech, Yucaipa, CA, USA) was used to ensure embedding of the teeth vertically in acrylic resin (Caulk Orthodontic Resin, Dentsply Caulk, York, PA, USA), which was poured into polyvinyl chloride (PVC) cylinders with a dimension of $25 \mathrm{~mm}$ diameter and $40 \mathrm{~mm}$ height. Following resin polymerization, the teeth were retrieved from the acrylic resin and the wax was eliminated under a steam cleaner. Consequently, the spaces of the melted wax were filled with the polyvinyl silicone impression material (Imprint, 3M ESPE, Seefeld, Germany) to imitate periodontal ligaments (PDL). The teeth were then inserted back into the sockets and excess materials were removed using a blade \#11. One investigator performed PDL simulation and teeth embedding in acrylic resin.

All teeth were instrumented using the ProFile rotary system (Dentsply Tulsa Dental, Tulsa, OK, USA) up to a size 40, $1 \mathrm{~mm}$ from the apex using the crown-down technique. The canals were irrigated with $2.5 \% \mathrm{NaOCl}$ solution. Paper points were used to dry the canals before obturation with a single cone of the corresponding size of gutta percha with the use of a resin-based sealer (AH Plus, DENTSPLY DeTrey GmbH, Konstanz, Germany). For 1 week, the teeth were stored in distilled water at $37^{\circ} \mathrm{C}$ in sealed plastic containers. One investigator performed the endodontic treatment of all the teeth. Figure 1 shows the schematic representation of the groups.
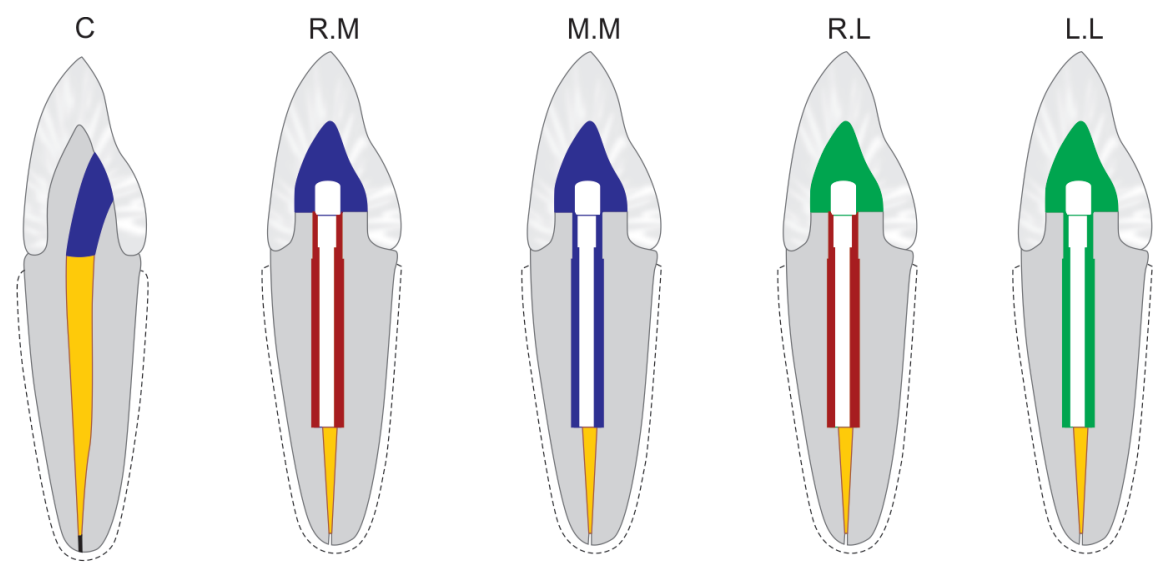

MultCore LuxaCore RelyX

Fig. 1: Schematic representation of the five groups: C, control group, access cavity was restored with Multicore; RM, RelyX Unicem for fiber post luting and Multicore for core buildup; MM, Multicore for fiber post luting and core buildup; RL, RelyX Unicem for fiber post luting and LuxaCore for core buildup; LL, LuxaCore for fiber post luting and core buildup 


\section{Group C: Control Group}

Access cavities of the canals were cleaned of gutta percha up to the CEJ, etched with $37 \%$ phosphoric acid gel for 15 seconds (Total Etch, Ivoclar Vivadent, Schaan, Liechtenstein), washed with water, dried with air and cotton pellets. The EXCITE F DSC (Ivoclar Vivadent) bonding agent was applied, air-thinned, and light-cured for 10 seconds. MultiCore composite resin (MultiCore Flow; Ivoclar Vivadent) was used to fill the access cavities and light-cured for 10 seconds.

\section{Experimental Groups}

A transparent matrix was made over the crown by using a thermovacuum machine to record the dimensions of the crown, to be used for the composite core buildup. The teeth were mounted perpendicularly to the root axis and decoronated at $2 \mathrm{~mm}$ above the CEJ using a low-speed diamond saw machine (Isomet 2000; Buehler Ltd., Lake Bluff, NY, USA) under a water-cooling system. Post space preparation was carried out using a universal drill, size 0 and size 1, respectively (RelyX Fiber Post, 3M ESPE), to remove gutta percha and prepare the canals, leaving a minimum of 4 $\mathrm{mm}$ of gutta percha to ensure apical seal for all experimental specimens. The post spaces were washed with distilled water and dried using paper points.

\section{Group RM: RelyX Unicem-MultiCore.}

The fiber posts (RelyX Fiber Post, size 1; 3M ESPE) were cemented by self-adhesive cements (RelyX Unicem, 3M ESPE) using elongation tips into the posts' spaces. The excess cement materials were removed followed by 40 seconds of light curing. The coronal dentin was etched and the bonding agent was applied as in group C. MultiCore composite resin was applied for core buildup with the corresponding transparent matrix for each tooth and light-cured for 10 seconds.

\section{Group MM: MultiCore-MultiCore}

The canal and coronal dentin were etched and the bonding agent was applied as in group C. Excess bonding materials were removed from the post spaces using paper points. MultiCore composite resin was applied into the posts' spaces using intracanal tips. The fiber posts were immediately inserted and light-cured for 20 seconds following the manufacturer's recommendations. MultiCore composite resin was applied for core buildup as in group RM.

\section{Group RL: RelyX Unicem-LuxaCore}

The fiber posts were cemented and the coronal dentin were etched as in group RM. The bonding agent (LuxaBond-Total Etch; DMG, Hamburg, Germany) was applied to the coronal dentin following the manufacturer's instructions. LuxaCore composite resin (LuxaCore Z-Dual; DMG, Hamburg, Germany) was applied for core buildup with the corresponding transparent matrix for each tooth and light-cured for 20 seconds.

\section{Group LL: LuxaCore-LuxaCore}

The canal and coronal dentin were etched as in group MM. The bonding agent (LuxaBond-Total Etch; DMG) was applied on the canal and the coronal dentin following the manufacturer's instructions. LuxaCore composite resin (LuxaCore Z-Dual; DMG) was applied into the post's space using the intracanal tip. The fiber posts were immediately inserted and light-cured for 20 seconds. LuxaCore composite resin was applied for core buildup as in group RL.

\section{Crown Restoration}

Preparation of all teeth was performed for all-ceramic crowns with round-shoulder margins of $1.2 \mathrm{~mm}$ circumferentially. Margins of the experimental groups were placed $2 \mathrm{~mm}$ apical to the level of the core material to attain the ferrule effect. All teeth were restored with zirconium crowns (Multi-Layered, High Translucent, KATANA Zirconia; Kuraray Noritake Dental, Inc., Tokyo, Japan). The crowns were cemented using self-adhesive cements (RelyX Unicem; 3M ESPE). Luting of the fiber posts, core buildup, teeth preparation, and crown cementations were performed by one investigator.

\section{Loading Protocol}

All specimens were subjected to thermocycling (THE-1100 Thermocycler; SD Mechatronik Gmbh, Feldkirchen-Westerham, Germany) for 6,000 thermal cycles at $5-55^{\circ} \mathrm{C}$ with 60 seconds dwell time in each baths and 10 seconds transferring time. Loading of the specimens was achieved using a universal testing machine at $0.5 \mathrm{~mm} /$ minute cross-speed (Instron 5965 Material Testing System; Instron Corp., High Wycombe, UK) until failure. The maximum failure load of each specimen was recorded in Newton and the mean of each group was calculated. A steel piston $(3 \mathrm{~mm} \times 1 \mathrm{~mm}$, rectangular) was used to apply force at $135^{\circ} \mathrm{C}$ to the palatal surface; a tin foil of $0.3 \mathrm{~mm}$ thickness was placed on the palatal surfaces of the crowns to minimize excessive stress concentrations, as shown in Figure 2.

\section{Evaluation of the Mode of Failure}

The mode of failure was analyzed under a digital microscope $(\mathrm{KH}-$ 7700, HIROX EUROPE, Tokyo, Japan). Fracture above or at the crestal bone level was considered a restorable failure, whereas below the crestal bone level was considered a nonrestorable failure. At the end of the experiment, all the specimens were discarded in a medical waste container.

\section{Statistical Analysis}

The statistical analysis of the fracture resistance data was performed using one-way ANOVA followed by a Games-Howell multiple comparison test (SPSS 25.0; Chicago, IL, USA). The level of significance was set at 0.05 .

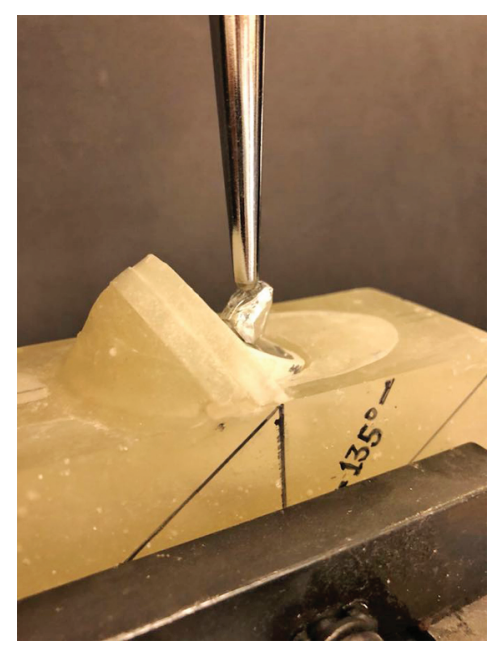

Fig. 2: Load application on a specimen: The load was applied at $135^{\circ}$ to the palatal surface; a tin foil was placed on the crowns to minimize excessive stress concentrations 


\section{Results}

The highest fracture resistance was found in the control group, $613 \pm 143 \mathrm{~N}$, followed by the RM group, $448 \pm 68 \mathrm{~N}$; the LL group, $406 \pm 109 \mathrm{~N}$; the RL group, $366 \pm 47 \mathrm{~N}$; and the MM group, $362 \pm$ $40 \mathrm{~N}$, respectively (Fig. 3). The ANOVA and Games-Howell multiple comparison tests (Table 1) showed that the fracture resistance of the control group (C group) was significantly higher than that of the experimental groups $(p<0.05)$. However, there was no significant difference among the experimental groups $(p>0.05)$.

All the specimens failed with root fractures below the level of the simulated crestal bone and were considered nonrestorable failures. The fractures extended from the ferrule effect on the palatal aspects toward the labial surfaces of the roots obliquely and confined to the cervical thirds of the root, as shown in Figure 4.

\section{Discussion}

In the current study, two dual-cure composite core materials were used-MultiCore and LuxaCore-and they were also used as post-luting agents and compared to a self-adhesive RelyX Unicem resin cement to assess their influence on fracture resistance of endodontically treated maxillary central incisor teeth restored with zirconium crowns. The results showed a significant difference between the control group and the experimental groups. However, no significant difference was found among the experimental groups. Therefore, the null hypothesis, which was no significant difference between the materials used for fiber post luting and core buildup, was accepted.

In the control group with only the access cavities of root canal treatment filled with MultiCore and restored with crowns, the presence of the coronal tooth structure demonstrated a significant role in fracture resistance. This is a fact that has been well-recognized in the literature by in vivo and in vitro studies. ${ }^{34-36}$ However, there was no significant difference among the experimental groups. Accordingly, the materials used for fiber post luting and core buildup did not make any significant difference in fracture resistance of the ETT. Previous clinical ${ }^{37,38}$ and laboratory studies $^{28,32,39}$ have reported similar results when comparing different resin materials for fiber post luting and core buildup. A possible explanation for this finding is the presence of the ferrule effect and crown restoration, which might conceal the influence of the different restorative methods. ${ }^{40}$ The crown under obliquely directed loading concentrates the stresses to the tooth structure at the ferrule effect site more than at the post and core system, which has been reported in photoelastic and finite-element studies. ${ }^{41,42}$ Nevertheless, the placement of crown restoration was essential to represent the clinical situation.

While comparing the different composite core materials (MultiCore and LuxaCore), the current study did not find any significant difference between the two materials. To the authors' knowledge, no previous study compared the same materials for fracture resistance when used for post luting. Nevertheless, an in vitro study has revealed no significant difference in fracture resistance between MultiCore and LuxaCore when used for core buildup. ${ }^{43}$ A retrospective clinical study with an 8 -year observation period has reported no significant difference between LuxaCore and a hybrid light-cure composite for core buildup. ${ }^{37}$ When the same material was used for post luting and core buildup (MM and LL groups) to establish a secondary monobloc restoration, the fracture resistance was not significantly different from the

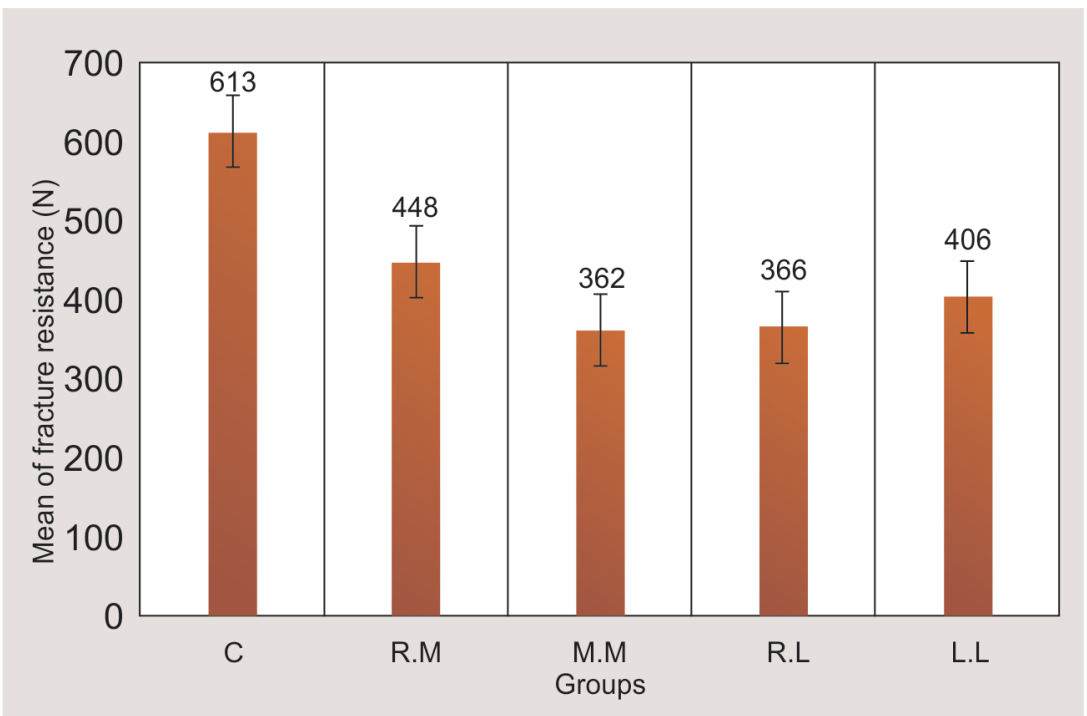

Fig. 3: Mean of fracture resistance in Newtons (N) of each group

Table 1: ANOVA and multiple comparison tests

\begin{tabular}{|c|c|c|c|c|c|c|c|c|c|c|c|}
\hline \multirow[b]{2}{*}{ Group } & \multirow[b]{2}{*}{$N$} & \multirow[b]{2}{*}{ Mean } & \multirow[b]{2}{*}{$S D$} & \multirow[b]{2}{*}{ ANOVA $p$ value } & \multicolumn{2}{|c|}{ 95\% confidence interval for mean } & \multicolumn{5}{|c|}{ Multiple comparison test } \\
\hline & & & & & Lower bound & Upper bound & $C$ & $R M$ & $M M$ & $R L$ & $L L$ \\
\hline C & 10 & 613 & 143 & 0.000000 & 510 & 715 & 1.000 & & & & \\
\hline RM & 10 & 448 & 68 & & 399 & 496 & 0.002 & 1.000 & & & \\
\hline MM & 10 & 362 & 40 & & 334 & 391 & 0.000 & 0.234 & 1.000 & & \\
\hline $\mathrm{RL}$ & 10 & 366 & 47 & & 332 & 400 & 0.000 & 0.274 & 1.000 & 1.000 & \\
\hline LL & 10 & 406 & 109 & & 328 & 484 & 0.000 & 0.841 & 0.815 & 0.859 & 1.000 \\
\hline
\end{tabular}




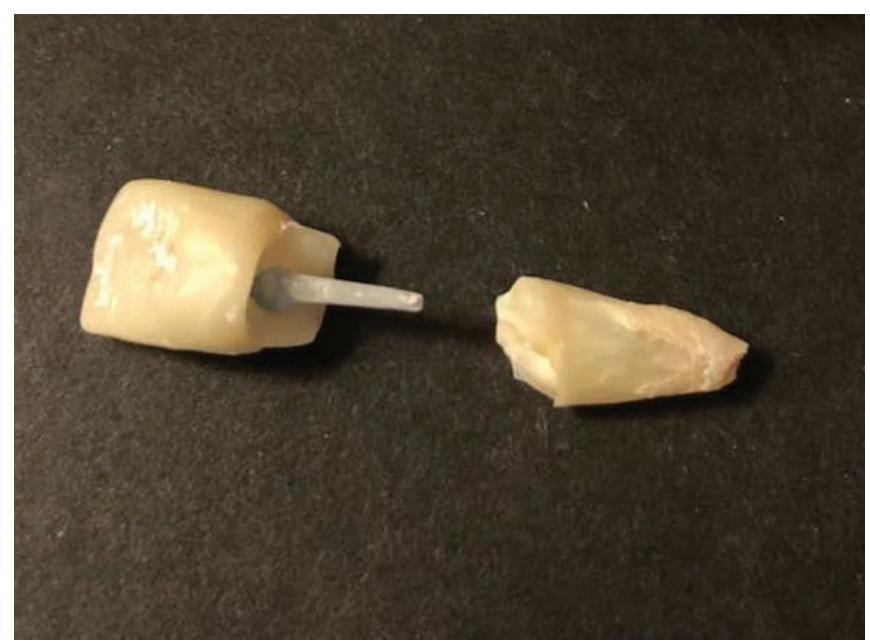

Fig. 4: Fractured specimen: the fracture extended from the ferrule effect on the palatal aspect toward the root labial surface obliquely

use of the self-adhesive resin cement for post luting (RM and RL groups). This is in agreement with the 4-year prospective clinical study by Juloski et al., which has reported that the failure risk of endodontically treated premolars was not influenced by the use of the same resin composite material for fiber post luting and core buildup. ${ }^{38}$ Similarly, an in vitro study by Kim and Lee showed that the use of a dual-cure composite core material (LuxaCore) for both fiber post luting and core buildup did not enhance the fracture strength and was not significantly different than the use of the conventional method. ${ }^{39}$ Naumann et al. found no significant difference in fracture resistance between the conventional method and the use of either a self-adhesive resin cement or a composite core material for both post luting and core buildup. ${ }^{28}$ Consequently, with the presence of a ferrule effect and a crown restoration, the strength of an endodontically treated tooth does not depend on the choice of the restorative material. However, Bitter et al. have reported contradictory results with the use of resin cement for core buildup in comparison to the different combinations of materials. They reported lower fracture resistance with the use of resin cement for core buildup, and was attributed to the low mechanical properties and increased water sorption of the resin cement as compared to the core buildup materials. ${ }^{28,32,44}$

The use of dual-cure composite core materials for fiber post luting has been investigated with regard to bond strength to the intracanal dentin. In an in vitro study, Boschian Pest et al. found that push-out bond strength was higher when luting fiber posts with composite resin than luting with resin cements. ${ }^{45}$ Bitter et al. investigated the influence of the adhesive technique (self-etch or etch-and-rinse) on the bond strength of four core materials used for luting fiber posts and revealed no difference in bond strength; all materials exhibited a homogeneous hybrid layer and adequate penetration into the dentinal tubules. ${ }^{46}$ However, Ferrari et al., in an in vitro study, have found that using high filler luting agents was associated with increased polymerization stress; it decreased push-out bond strength and increased interfacial nanoleakage. ${ }^{47}$

The recorded fracture resistance of the experimental groups demonstrated higher values than the reported intraoral maximum forces exerted by adult males at the maxillary incisor tooth (146 \pm $44 \mathrm{~N}) .{ }^{48}$ Therefore, the fracture resistance of the maxillary central incisors would not be affected by the choice of material used for post luting and core buildup in the presence of a 2-mm ferrule effect. The findings of the current study support the use of the same material for post luting and core buildup for only the simplification of the clinical procedure, since no enhancement of fracture resistance was reported, compared to the conventional method.

All the specimens had the same mode of failure, with fracture extending from the ferrule effect on the palatal aspect to the labial root surface obliquely and were confined to the cervical thirds of the roots, which is consistent with several previous studies. ${ }^{36,41-43,49}$ However, this mode of failure does not represent the commonly encountered clinical failure. ${ }^{36}$ The possible explanation of this finding is that the teeth were mounted in the acrylic resin with a simulated PDL, which does not replicate the behavior of the alveolar bone and PDL under occlusal forces. Consequently, as stated by Assif et al., the static loading concentrates the forces at the gingival margins of the crown, with the fulcrum located in the simulated alveolar crest area. ${ }^{41}$

There are some limitations to the current study. Although the teeth were subjected to thermocycling, static loading does not represent the intraoral forces applied on the teeth. However, fatigue loading of the specimens would represent the clinical situation. Moreover, only central incisors were used in this study and the findings cannot be generalized to other types of teeth. In addition, omission of the ferrule effects and crown restorations on the specimens could provide a more accurate evaluation of the influence of the different restorative methods. Ultimately, clinical studies with a large sample size should provide stronger evidence on the effectiveness of the monobloc system on the fracture resistance of ETT.

\section{Conclusion}

The use of dual-cure composite core materials for fiber post luting did not differ significantly from the self-adhesive resin cement in regard to fracture resistance of endodontically treated maxillary central incisors with a 2-mm ferrule effect and crown restoration. However, it would simplify the clinical procedure.

\section{Clinical Significance}

The use of the same dual-cure composite core materials for fiber post luting and core buildup would simplify the clinical procedure without enhancement of the fracture resistance of ETT.

\section{References}

1. Williams JV, Williams LR. Is coronal restoration more important than root filling for ultimate endodontic success? Dent Update 2010;37(3):187-193. DOI: 10.12968/denu.2010.37.3.187.

2. Morgano SM, Rodrigues AH, Sabrosa CE. Restoration of endodontically treated teeth. Dent Clin North Am 2004;48(2):397-416. DOI: 10.1016/j. cden.2003.12.011.

3. Marchi GM, Paulillo LA, Pimenta LA, et al. Effect of different filling materials in combination with intraradicular posts on the resistance to fracture of weakened roots. J Oral Rehabil 2003;30(6):623-629. DOI: 10.1046/j.1365-2842.2003.00990.x.

4. Tjan AH, Whang SB. Resistance to root fracture of dowel channels with various thicknesses of buccal dentin walls. J Prosthet Dent 1985;53(4):496-500. DOI: 10.1016/0022-3913(85)90633-x.

5. Zogheib LV, Saavedra Gde S, Cardoso PE, et al. Resistance to compression of weakened roots subjected to different root reconstruction protocols. J Appl Oral Sci 2011;19(6):648-654. DOI: 10.1590/s1678-77572011000600018. 
6. Gutmann JL. The dentin-root complex: anatomic and biologic considerations in restoring endodontically treated teeth. J Prosthet Dent 1992;67(4):458-467. DOI: 10.1016/0022-3913(92)90073-j.

7. Goracci C, Ferrari M. Current perspectives on post systems: a literature review. Aust Dent J 2011;56(1):77-83. DOI: 10.1111/j.18347819.2010.01298.x.

8. Morgano SM, Brackett SE. Foundation restorations in fixed prosthodontics: current knowledge and future needs. J Prosthet Dent 1999;82(6):643-657. DOI: 10.1016/s0022-3913(99)70005-3.

9. Maccari PC, Cosme DC, Oshima HM, et al. Fracture strength of endodontically treated teeth with flared root canals and restored with different post systems. J Esthet Restor Dent 2007;19(1):30-36. DOI: 10.1111/j.1708-8240.2006.00060.x; discussion 7.

10. Freedman GA. Esthetic post-and-core treatment. Dent Clin North Am 2001;45(1):103-116.

11. Figueiredo FED, Martins-Filho PRS, Faria-e-Silva AL. Do metal post-retained restorations result in more root fractures than fiber post-retained restorations? A systematic review and meta-analysis. J Endod 2015;41(3):309-316. DOI: 10.1016/j.joen.2014.10.006.

12. Bateman G, Ricketts DN, Saunders WP. Fibre-based post systems: a review. Br Dent J 2003;195(1):43-48. DOI: 10.1038/sj.bdj.4810278; discussion 37.

13. Hayashi M, Takahashi Y, Imazato S, et al. Fracture resistance of pulpless teeth restored with post-cores and crowns. Dent Mater 2006;22(5):477-485. DOI: 10.1016/j.dental.2005.03.017.

14. Standlee JP, Caputo AA, Hanson EC. Retention of endodontic dowels: effects of cement, dowel length, diameter, and design. J Prosthet Dent 1978;39(4):400-405. DOI: 10.1016/s0022-3913(78)80156-5.

15. Adanir N, Belli S. Stress analysis of a maxillary central incisor restored with different posts. Eur J Dent 2007;1(2):67-71. DOI: 10.1055/s-00391698316.

16. Newman MP, Yaman P, Dennison J, et al. Fracture resistance of endodontically treated teeth restored with composite posts. J Prosthet Dent 2003;89(4):360-367. DOI: 10.1067/mpr. 2003.75.

17. Sirimai S, Riis DN, Morgano SM. An in vitro study of the fracture resistance and the incidence of vertical root fracture of pulpless teeth restored with six post-and-core systems. J Prosthet Dent 1999;81(3):262-269. DOI: 10.1016/s0022-3913(99)70267-2.

18. Bonfante $G$, Kaizer OB, Pegoraro LF, et al. Fracture strength of teeth with flared root canals restored with glass fibre posts. Int Dent J 2007;57(3):153-160. DOI: 10.1111/j.1875-595x.2007.tb00118.x.

19. Biacchi GR, FLBd A, França FMG, et al. Mechanical properties of flared root canals restored with fiber post and chemically activated resin: Study using push-out bond strength and fracture load tests. J Adhes Sci Technol 2016;30(13):1441-1452. DOI: 10.1080/01694243.2016.1147126.

20. Ferrari M, Vichi A, Garcia-Godoy F. Clinical evaluation of fiberreinforced epoxy resin posts and cast post and cores. Am J Dent 2000;13(Spec No):15B-18B.

21. Akkayan B, Gulmez T. Resistance to fracture of endodontically treated teeth restored with different post systems. J Prosthet Dent 2002;87(4):431-437. DOI: 10.1067/mpr.2002.123227.

22. Dallari $A$, Rovatti $L$. Six years of in vitro/in vivo experience with composipost. Compendium of continuing education in dentistry (Jamesburg, NJ: 1995) supplement. 1996(20):S57-S63.

23. Tay FR, Pashley DH. Monoblocks in root canals: a hypothetical or a tangible goal. J Endod 2007;33(4):391-398. DOI: 10.1016/j. joen.2006.10.009.

24. Vichi A, Carrabba M, Goracci C, et al. Extent of cement polymerization along dowel space as a function of the interaction between adhesive and cement in fiber post cementation. J Adhes Dent 2012;14(1):51-57. DOI: 10.3290/j.jad.a21849.

25. Perdigão J. Restoration of Root Canal-Treated Teeth: An Adhesive Dentistry Perspective. Springer; 2015.

26. Monticelli F, Osorio R, Mazzitelli C, et al. Limited decalcification/ diffusion of self-adhesive cements into dentin. J Dent Res 2008;87(10):974-979. DOI: 10.1177/154405910808701012.
27. Yoldas $\mathrm{O}$, Alaçam T. Microhardness of composites in simulated root canals cured with light transmitting posts and glass-fiber reinforced composite posts. J Endod 2005;31(2):104-106. DOI: 10.1097/01. don.0000133160.08600.47.

28. Naumann M, Sterzenbach $G$, Rosentritt $M$, et al. Self-adhesive cements as core build-ups for one-stage post-endodontic restorations? Int Endod J 2011;44(3):195-202. DOI: 10.1111/j.1365-2591. 2010.01797.x.

29. O'Keefe KL, Powers JM. Adhesion of resin composite core materials to dentin. Int J Prosthod 2001;14(5):451-456.

30. Kajihara H, Suzuki S, Minesaki Y, et al. Effect of filler loading on resin cement bonding to silanized buildup composites. Am J Dent 2005;18(2):109-112.

31. Naumann M, Kiessling S, Seemann R. Treatment concepts for restoration of endodontically treated teeth: a nationwide survey of dentists in Germany. J Prosthet Dent 2006;96(5):332-338. DOI: 10.1016/j.prosdent.2006.08.028.

32. Naumann M, Sterzenbach G, Rosentritt M, et al. In vitro performance of self-adhesive resin cements for post-and-core build-ups: influence of chewing simulation or 1-year storage in $0.5 \%$ chloramine solution. Acta Biomater 2010;6(11):4389-4395. DOI: 10.1016/j. actbio.2010.05.023.

33. Monaco C, Llukacej A, Baldissara P, et al. Zirconia-based versus metal-based single crowns veneered with overpressing ceramic for restoration of posterior endodontically treated teeth: 5-year results of a randomized controlled clinical study. J Dent 2017;65:56-63. DOI: 10.1016/j.jdent.2017.07.004.

34. Lima AFD, Spazzin AO, Galafassi D, et al. Influence of ferrule preparation with or without glass fiber post on fracture resistance of endodontically treated teeth. J Appl Oral Sci 2010;18(4):360-363. DOI: 10.1590/s1678-77572010000400007.

35. Guzy GE, Nicholls JI. In vitro comparison of intact endodontically treated teeth with and without endo-post reinforcement. J Prosth Dent 1979;42(1):39-44. DOI: 10.1016/0022-3913(79)90328-7.

36. Tan PLB, Aquilino SA, Gratton DG, et al. In vitro fracture resistance of endodontically treated central incisors with varying ferrule heights and configurations. J Prosthet Dent 2005;93(4):331-336. DOI: 10.1016/j.prosdent.2005.01.013.

37. Signore A, Benedicenti S, Kaitsas V, et al. Long-term survival of endodontically treated, maxillary anterior teeth restored with either tapered or parallel-sided glass-fiber posts and full-ceramic crown coverage. J Dent 2009;37(2):115-121. DOI: 10.1016/j.jdent.2008. 10.007 .

38. Juloski J, Fadda G, Monticelli F, et al. Four-year survival of endodontically treated premolars restored with fiber posts. J Dent Res 2014;93(7):52S-58SS. DOI: 10.1177/0022034514527970.

39. Kim YH, Lee JH. Influence of modification in core building procedure on fracture strength and failure patterns of premolars restored with fiber post and composite core. J Adv Prosthodont 2012;4(1):37-42. DOI: 10.4047/jap.2012.4.1.37.

40. Heydecke G, Butz F, Hussein A, et al. Fracture strength after dynamic loading of endodontically treated teeth restored with different postand-core systems. J Prosthet Dent 2002;87(4):438-445. DOI: 10.1067/ mpr.2002.123849.

41. Assif D, Oren E, Marshak BL, et al. Photoelastic analysis of stress transfer by endodontically treated teeth to the supporting structure using different restorative techniques. J Prosthet Dent 1989;61(5):535-543. DOI: 10.1016/0022-3913(89)90272-2.

42. Ichim I, Kuzmanovic D, Love R. A finite element analysis of ferrule design on restoration resistance and distribution of stress within a root. Int Endod J 2006;39(6):443-452. DOI: 10.1111/j.13652591.2006.01085.x.

43. Panitiwat P, Salimee P. Effect of different composite core materials on fracture resistance of endodontically treated teeth restored with FRC posts. J Appl Oral Sci 2017;25(2):203-210. DOI: 10.1590/167877572016-0306.

44. Bitter K, Schubert A, Neumann K, et al. Are self-adhesive resin cements suitable as core build-up materials? analyses of maximum 
load capability, margin integrity, and physical properties. Clin Oral Investig 2016;20(6):1337-1345. DOI: 10.1007/s00784-0151623-0.

45. Boschian Pest L, Cavalli G, Bertani P, et al. Adhesive post-endodontic restorations with fiber posts: push-out tests and SEM observations. Dent Mater 2002;18(8):596-602. DOI: 10.1016/s0109-5641(02)00003-9.

46. Bitter K, Gläser C, Neumann K, et al. Analysis of resin-dentin interface morphology and bond strength evaluation of core materials for one stage post-endodontic restorations. PLoS ONE 2014;9(2):e86294. DOI: 10.1371/journal.pone.0086294.
47. Ferrari M, Carvalho CA, Goracci C, et al. Influence of luting material filler content on post cementation. J Dent Res 2009;88(10):951-956. DOI: $10.1177 / 0022034509342851$.

48. Ferrario V, Sforza C, Serrao G, et al. Single tooth bite forces in healthy young adults. J Oral Rehabil 2004;31(1):18-22. DOI: 10.1046/j.0305182x.2003.01179.x.

49. Qing $H$, Zhu Z, Chao $Y$, et al. In vitro evaluation of the fracture resistance of anterior endodontically treated teeth restored with glass fiber and zircon posts. J Prosthet Dent 2007;97(2):93-98. DOI: 10.1016/j.prosdent.2006.12.008. 